\title{
Multi-Robot Localization System using an Array of LEDs and LDR Sensors
}

\author{
Israa Sabri Abdulameer AL-Forati \\ Electrical Engineering Department \\ University of Basrah \\ Basrah, Iraq
}

\author{
Abdulmutallab Rashid \\ Electrical Engineering Department \\ University of Basrah \\ Basrah, Iraq
}

\begin{abstract}
a new positioning system for indoor multi-robot localization is proposed. This system solves the problem of localization by using an array of Light Emitting Diodes (LEDs) distributed uniformly in the environment. The localization is achieved by collecting the information from a group of Light Dependent Resistor (LDR) sensors with which the robot is equipped. The binary search algorithm is used to reduce the time of the localization process by controlling the lights of the LED array. The minimum bounded circle algorithm is used to draw a virtual circle from the information collected by the LDR sensors and the center of this circle represents the robot's location. This algorithm can be implemented in a multi-robot system when the main control unit can distinguish among the LDR sensors' information. In the case of unknown information, the K-means Clustering algorithm is used to separate this information into clusters. Each cluster can be used to estimate the location of one robot. The suggested system is simulated and practically implemented in an environment with $(32 * 32)$ arrays of LEDs. The simulation and experimental results of this system show good performance in the localization process.
\end{abstract}

\section{General Terms}

Light Emitting Diode (LEDs), Light Dependent Resistor (LDR), multi-robot system.

\section{Keywords}

Localization system, Binary search algorithm, Minimum bounded circle algorithm.

\section{INTRODUCTION}

Now a day multi-mobile robot is used in many fields such as path planning and obstacle avoidance, object tracking, inspection, surveillance and multi-mobile robot formation [13]. A localization technique is one of the essential requirements for a multi-robot system which is necessary to determine the location and direction of the mobile robot based on one of the localization algorithms and earlier knowledge represents by the environment map or known beacons. It is difficult to perform any task by any mobile robot without knowing its position in the environment [4]

Various localization techniques have been advanced for an indoor mobile robot. Surveys, in [5-9] including comparisons, of an indoor mobile robot localization method. Some applications based on IR systems using passive Landmark for localization of indoor mobile robots Was developed. The [10] Hagisonic StarGazer localization system for indoor mobile robots is usually using a passive landmark based on localization systems. Experimentally, this system was evaluated by [11], who found the system to be accurate, reliable, affordable, and robust. They similarly determined the orientation accuracy and position to be sensibly high based on their new data. [12] established an indoor localization system built on the Hagisonic Star-Gazer localization system. They also found some categories of deviations from the actual values and evaluated this system. They reduced the errors that happened during the training by using an adaptive extended Kalman filter and optimum planning of landmarks. Their experimental results confirmed to very good precision.

a localization system developed in [13] employed the same principle as the Hagisonic StarGazer localization system of reflective landmarks. Artificial landmarks were formed using films covered with IR light-reflecting solid. an IR signal emitter and detector are important mechanisms in a localization system built on active landmarks. A lot of methods employed an emitter as the active landmark. Some of them are used IR beacons [14] that only provided data about the bearing. Their system involved placing beacons in the environment with known positions. from these beacons, the signals were then sensed by a scanner device located on the highest of the robot chassis.

The accuracy and inexpensiveness of light-emitting diode (LED) sensors, along with their extended life expectancy, is valuable: lights are expected to continue for many years; moreover, the light is harmless to humans, unlike IR and lasers, and it does not overlap with the effects of electronic devices in the same environment. The foregoing features make LEDs a very suitable choice for use in localization scenarios. Practical experiments can test the light sensors with low-cost LEDs \& LDRs $[15,16]$.

\section{DESIGN AND IMPLEMENTATION THE INDOOR MULTI-ROBOT LOCALIZATION SYSTEM}

A new algorithm for indoor multi-robot localization is presented. It relies on estimating the position of the robot concerning the indoor environment. The localization is achieved using pairs of sensors: a group of LDRs fixed on the robot represents the first part and the second is represented by a matrix of white LEDs distributed uniformly in the environment. The binary search algorithm [17] is used to control the lighting of the matrix of LEDs and the data collected from the LDR sensors is directed to the central unit. The robot location is estimated by applying the minimum bounded circle algorithm [18] to the collected information. The procedure is used in this paper to estimate the location of each robot is similar to the procedure mentioned above when each robot has a different ID for communicating and sending the collected data to the main control unit. The problem occurs when the main control unit has not the intelligence to recognize the source of information received. To solve this problem and estimate the multi-robot locations, the k-means 
clustering algorithm [19] is used to isolate the received information into clusters. The center of each cluster represents the location of one robot when using the proposed method. Fig. 1 shows the multi-robot system with four robots each with four LDR sensors circling uniformly around the surrounding of that robot. The LED array is scanned column by column using the binary search algorithm and at each step, the LDR sensors on the robots check to sense any neighboring lighting LED. After completing the scan of all the columns, the process repeats on the rows of the labeled column. All the collected information is sent to the main control unit to estimate the location of each robot. The problem occurs when the main control does not know the reference of each information. The solving of this problem is done by using the k-Means Clustering Algorithm [19].

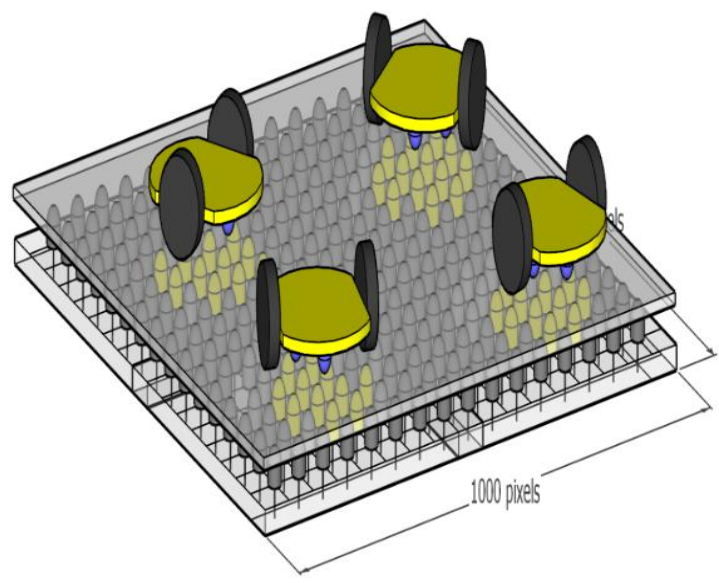

Fig 1: Multi-robot localization system.

The k-means clustering algorithm is a simple and perfect method to fragment a large group of data. This algorithm can separate the group of data to any number of clusters by repeating several trials to find the best number of clusters. In this work, the group of data represents the labeled LEDs that represent the LEDs sensed by the LDR sensors on the robots. Each sub-group of the labeled LEDs belongs to one robot. The objective of the k-means technique is to divide the labeled LEDs into k-clusters. The procedure for implementing this algorithm is:

(1) Randomly choose $\mathrm{K}$ locations on the environment to be the initial location of $\mathrm{N}$ Cluster (K robots).

(2) Separate the labeled LEDs into groups. The principle of separating is accomplished by determining the Euclidean distances ED according to equation (1) between each labeled LED and all the K points.

$$
E D=\sqrt{\sum_{i=1}^{n}\left(C_{i}-x_{i}\right)^{2}}
$$

Where $x i$ is the location of the labeled LED $i, n$ is the overall number of the labeled LEDs, and $\mathrm{Ci}$ is the location of the Cluster i.

The labeled LEDs are grouped into Clusters according to their distances from each cluster. Each labeled LED is added to the cluster with minimum distance.

(3) The center of each cluster (robot location) is computed by using centroid equation 2 .

Centroidpoint $i=\left(\frac{\left(\sum_{i=1}^{m} x_{i}\right)}{m}, \frac{\left(\sum_{i=1}^{m} y_{i}\right)}{m}\right)$
Where $\mathrm{m}$ is the numeral of the labeled LEDs sensors in cluster i.

(4) Use the computed centroid points as new locations for the cluster groups and repeat the above steps to rearrange the labeled LEDs among the clusters.

(5) Repeat steps 2 to 4 until all the labeled LEDs fixed in their clusters.

Fig.6. shows the suggested flow chart for working the kmeans clustering. Fig.7. shows the stages of executing the algorithm on individual labeled LEDs.

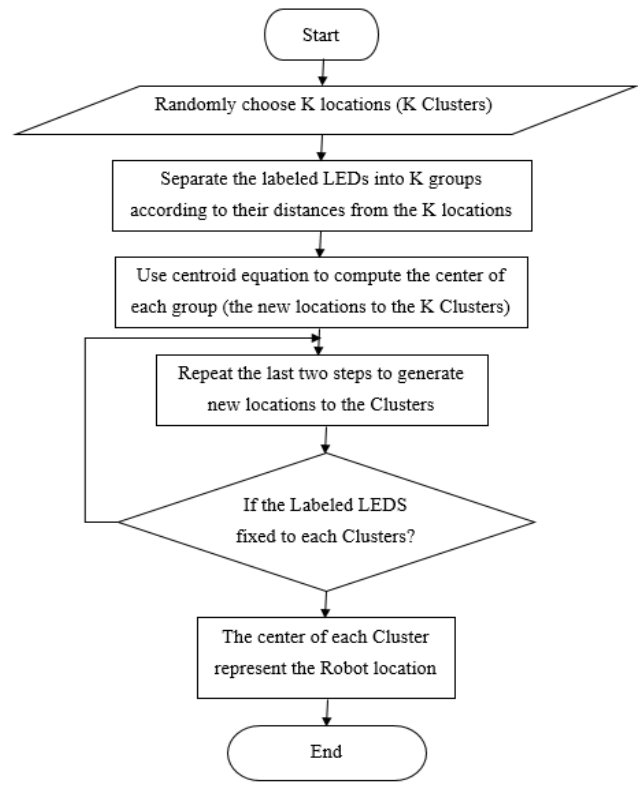

Fig 2: Flow chart for the k-means clustering algorithm.

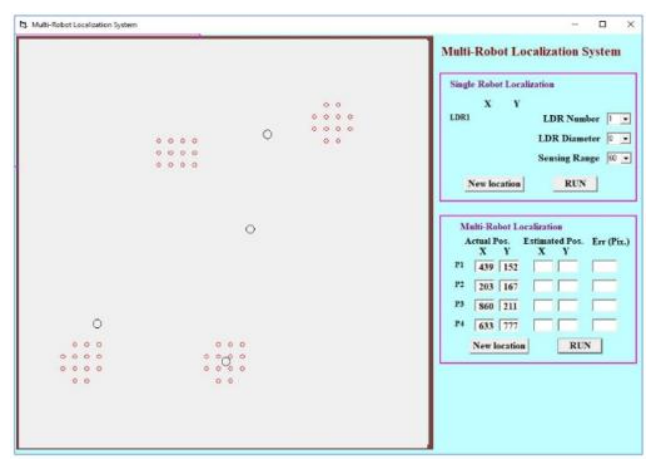

(a)

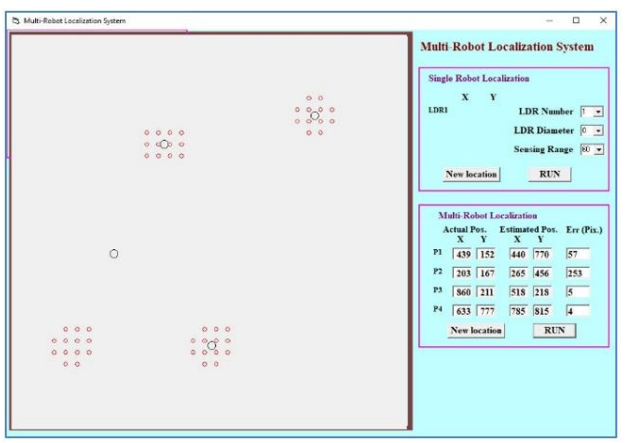

(b) 


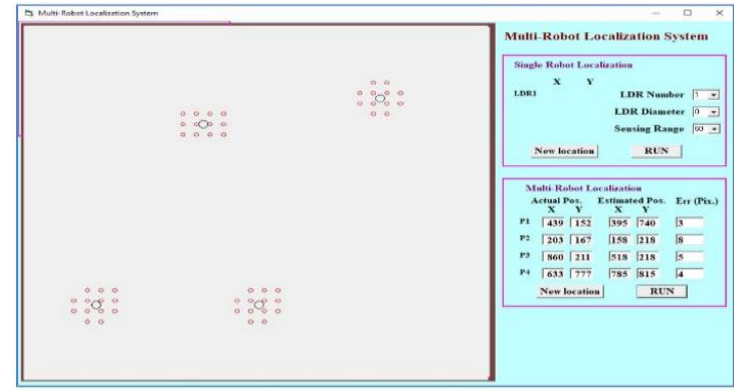

(c)

Fig 3: ( $a, b$ and c). Several iterations to reach the center of the robot.

\section{SIMULATION RESULTS}

In this paper, an original robotic localization scheme is validated by simulation. Simulations are performed for the multi-robot system with different numbers of LDR sensors (1, 2,3 , and 4). The simulations are repeated for 50 different topologies representing different robot locations, different sensing ranges for the LDR sensors and different distances between the LDR sensors. The dimensions of the environment used for these simulations are $1000 * 1000$ pixels with a $32 * 32$ LED matrix distributed uniformly. The system parameters used in these simulations are:

1. The number of LDR sensors on each robot.

2. Maximum sensing range of the LDR sensor.

3. Number of LEDs in the LED matrix.

The first group of simulations is used to show the implementation of the multi-robot localization using the kmean clustering algorithm. Figs. 4, 5, and 6 show the first group of simulations for the localization system on a robot with 40,60, and 80-pixel LDR sensing range respectively.

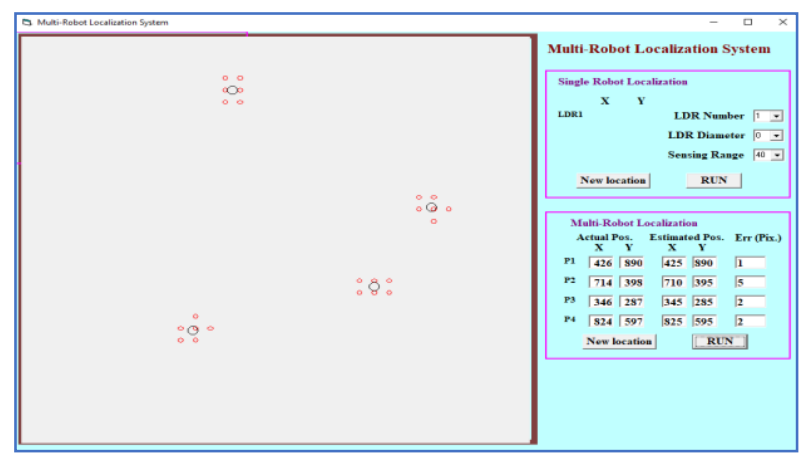

Fig 4: Simulation for multi-robot localization system (40pixel LDR sensing range).

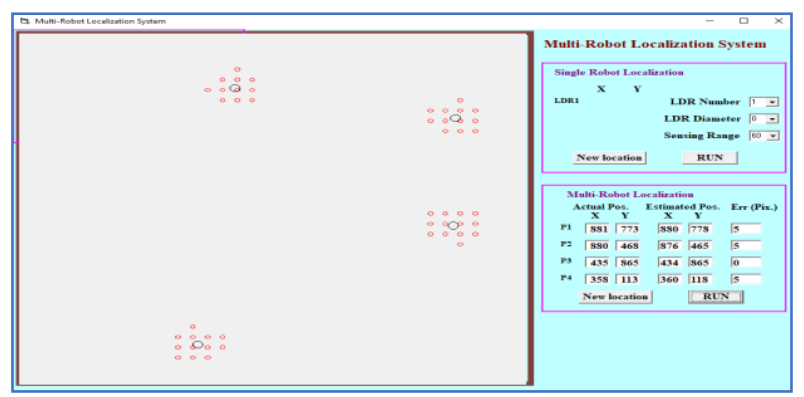

Fig 5: Simulation of multi-robot localization system (60pixel LDR sensing range).

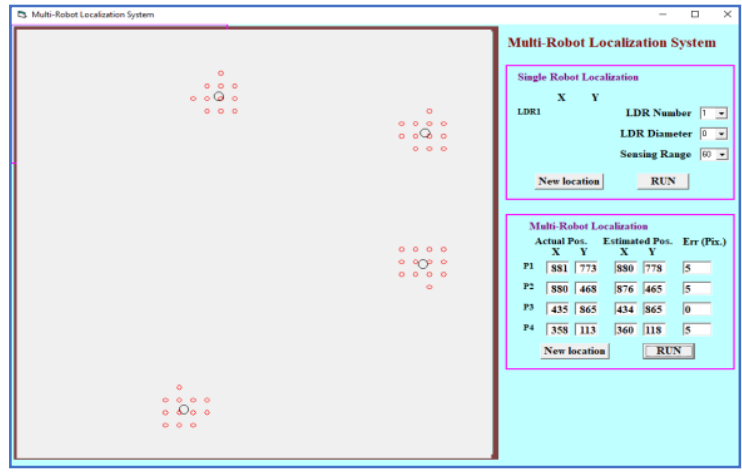

Fig 6: Simulation for multi-robot localization system (80pixel LDR sensing range).

At first, the simulation is used to compare the average of the iterations of the k-mean clustering with respect to the LDR receiver sensing range (Fig. 7). By repeating this simulation 50 times, the result shows that as the sensing range of the LDR receiver sensor is increased, the average repeated iteration numbers also increase.

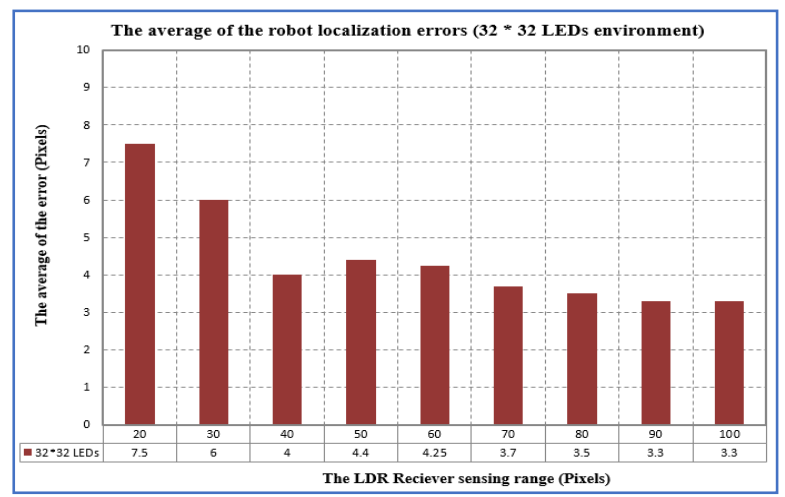

Fig 7: Average iteration of the k-means clustering algorithm in robot localization.

The same comparison procedure in Fig.7 is repeated for the multi-robot localization (Fig. 8). This comparison is used to show the relationship between the average error and the LDR sensing range. The result shows that as the LDR sensing range increases the average of the error decreases.it is shown in the table.1. that as the sensing range of the LDR sensor is increase the speed of the localization also increase because the scan process take more time ,also the average number of iterations is decrease when the sensing range is increase, on the other hand the average error (pixels) is is decrease when the sensing range is increase because the distance between the LEDs is decrease then the error is became minimum.

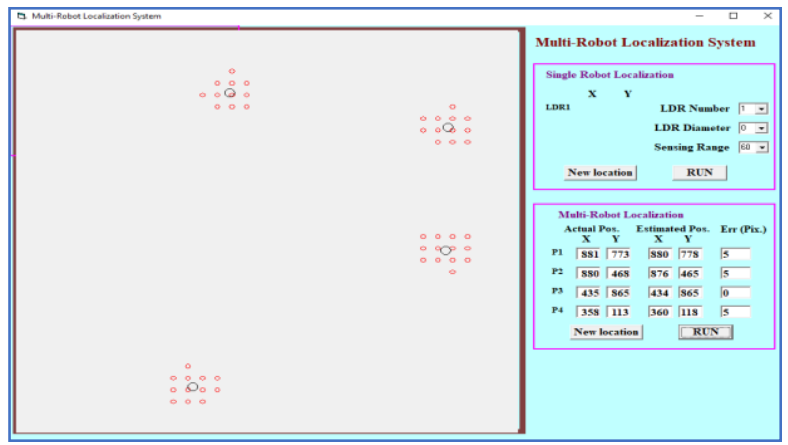




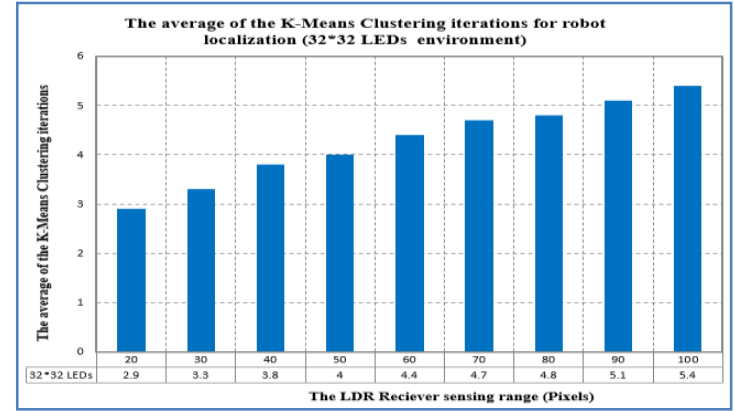

Fig 8: Average of the errors occurring in the multi-robot localization.

Table 1. Effect of various LDR sensing range on localization environment.

\begin{tabular}{|c|c|c|c|}
\hline \multirow{2}{*}{\begin{tabular}{c}
\multirow{2}{*}{$\begin{array}{c}\text { Sensing } \\
\text { range(pixels) }\end{array}$} \\
\cline { 2 - 4 }
\end{tabular}} & \multicolumn{3}{|c|}{ Localization technique properties in an environment (32*32) } \\
\hline 20 & 23 & $\begin{array}{c}\text { Average } \\
\text { error(pixels) }\end{array}$ & $\begin{array}{c}\text { Average Number of } \\
\text { iterations }\end{array}$ \\
\hline 40 & 34 & 61 & 12 \\
\hline 60 & 45 & 42 & 9 \\
\hline 80 & 57 & 39 & 7 \\
\hline 100 & 68 & 32 & 5 \\
\hline
\end{tabular}

\section{CONCLUSION}

An indoor robot localization system is introduced. This system solves the problem of localization by using an array of LEDs distributed uniformly in the environment. The localization process is simulated and tested on a multi-robot system with different numbers of LDR sensors $(1,2,3$, and 4 LDR sensors) and different sensing ranges (20 to 100 pixels). In the multi-robot system, the localization process is simulated and tested on a four-robot system with various numbers of environments and different sensing ranges. The simulation results show that the greatest sensing range is 20 pixels. Similarly, the results show that the average error that occurs in the robot localization is reduced as the LDR receiver sensing range increases. Moreover, the best case in simulation results is obtained when the sensing range is equal to 20 pixels.

\section{REFERENCES}

[1] A.T. Rashid, A. A. Ali, M. Frasca, and L. Fortuna (2013). Path planning with obstacle avoidance based on the visibility binary tree algorithm. Robotics and Autonomous Systems, 61(12), 1440-1449.

[2] Z. Y. Ibrahim, A. T. Rashid and A. F. Marhoon (2016). An algorithm for Path planning with polygon obstacle avoidance based on the virtual circle tangents. Iraq Journal Electrical and Electronic Engineering, 12(2), 221-234.

[3] Z. Y. Ibrahim, A. T. Rashid and A. F. Marhoon (2016). Prediction-Based Path Planning with Obstacle Avoidance in Dynamic Target Environment. Basrah Journal of Engineering Science, 16(2), 48-60.

[4] A. T. Rashid, M. Frasca, A, A, Ali, A. Rizzo and L. Fortuna (2015) Multi-robot localization and orientation estimation using robotic cluster matching algorithm. Robotics and Autonomous Systems, 63, 108-121.

[5] Mautz R, Tilch S (2011) Survey of optical indoor positioning systems. In:2011 international conference on indoor positioning and indoor navigation, pp 1-7. https

\section{://doi.org/10.1109/IPIN.2011.60719 25}

[6] Nuaimi KA, Kamel H (2011) A survey of indoor positioning systems and algorithms. In: 2011 international conference on innovations in information technology, pp 185-190. https ://doi.org/10.1109/INNOV ATIONS.2011.58938 13

[7] Koyuncu H, Yang SH (2010) A survey of indoor positioning and object locating systems. IJCSNS Int $\mathrm{J}$ Comput Sci Netw Secur 10(5):121-128

[8] Liu H, Darabi H, Banerjee P, Liu J (2007) Survey of wireless indoor positioning techniques and systems. IEEE Trans Syst Man Cybern Part C Appl Rev 37(6):1067-1080. https ://doi.org/10.1109/TSMCC .2007 .905750

[9] Desouza GN, Kak AC (2002) Vision for mobile robot navigation: a survey. IEEE Trans Pattern Anal Mach Intell 24(2):237-267. https ://doi.org/10.1109/34.98290 3

[10] robotshop: Hagisonic StarGazer RS robot localization system. http://www.robot shop.com/en/hagis onic-starg azer-rs-localization -syste m.html

[11] Ul-Haque I, Prassler E (2010) Experimental evaluation of a low-cost mobile robot localization technique for large indoor public environments. In: ISR 2010 (41st international symposium on robotics) and ROBOTIK 2010 (6thGerman conference on robotics), pp 1-7

[12] Oh JH, Kim D, Lee BH (2014) An indoor localization system for mobile robots using an active infrared positioning sensor. J Ind Intell Inf2(1):35-38

[13] Lee S (2009) Use of infrared light reflecting landmarks for localization. Ind Robot Int J 36(2):138-145. https ://doi.org/10.1108/01439910910932595

[14] Krejsa J, Vechet S (2012) Infrared beacons-based localization of mobile robot. Elektronika ir Elektrotechnika 117(1):17-22

[15] I. S. Alfurati and Abdulmuttalib T. Rashid (2018). Performance Comparison of Three Types of Sensor Matrices for Indoor Multi-Robot Localization. International Journal of Computer Applications (0975 8887), 181 (26), 22-29.

[16] I. S. Alfurati and Abdulmuttalib T. Rashid. An Efficient Mathematical Approach for an Indoor Robot Localization System. Iraqi Journal of Electrical and Electronic Engineering, 15 (2), 61-70, 2019.

[17] E. E. Odokuma and O. O. Owolabi (2016). An Indexed Method for Improving the Efficiency of the Binary Search Algorithm. International Journal of Advanced Research in Computer Science and Software Engineering, 6(5).

[18] I. S. Alfurati and Abdulmuttalib T. Rashid (2019)," Design and Implementation an Indoor Robot Localization System Using Minimum Bounded Circle Algorithm", The 8th International Conference on Modeling, Simulation and Applied Optimization (ICMSAO'2019)

[19] I. S. Alfurati, A. T. Rashid and Alaa Al-Ibadi (2019). IR sensors array for robot's localization using $\mathrm{K}$ means clustering algorithm. International Journal of Simulation Systems, Science \& Technology, 12.1-12.6. 